\title{
Características clínicas y factores pronósticos de la enfermedad meningocóccica: un estudio de serie de casos en Chile durante el brote 2012-2013
}

\author{
Isabel Matute, Andrea Olea, Darío López, Sergio Loayza, Manuel Nájera, Claudia González, \\ Lucy Poffald, Macarena Hirmas, Iris Delgado, Elena Pedroni, Tania Alfaro, Ana María Gormaz, \\ Gabriel Sanhueza, Pablo Vial, Jeannette Dabanch, Doris Gallegos y Ximena Aguilera
}

\section{Clinical features and prognostic factors of meningococcal disease: a case series study in Chile during the 2012-2013 outbreak}

Introduction: Meningococcal disease (MD) is a major global problem because of its case fatality rate and sequels. Since 2012 cases of serogroup W have increased in Chile, with nonspecific clinical presentation, high case fatality rate and serious consequences. Objective: To characterize the evolution and outcome of MD cases between January 2012 and March 2013 in Chile. Material and Methods: Case series considering149 MD cases of 7 regions. A questionnaire was applied and clinical records were reviewed, including individual, agent, clinical course and healthcare process variables. The analysis allowed to obtain estimates of the OR as likelihoodof dying. Results: $51.5 \%$ was meningococcemia, the case fatality rate reached $27 \%$, prevailing serogroup W (46.6\%). Factors that increased the probability of dying: $>$ age, belonging to indigenous people, having lived a stressful event, having diarrhea, impaired consciousness, cardiovascular symptoms, low oxygen saturation and low Glasgow coma scale score. Discussion: The case fatality rate exceeded normal levels and was higher in serogroup W. Increasing in this serogroup, associated to the increased presence of nonspecific symptoms or rapid progression to septicemia, hit a health system accustomed to more classic meningococcal disease presentation, which could partly explain the observed increased fatality rate.

Key words: Meningococcal disease, serogroup, case fatality rate, complications.

Palabras clave: Enfermedad meningocóccica, serogrupo, letalidad, complicaciones.

\section{Introducción}

L a enfermedad meningocóccica (EM) continúa siendo un importante problema de salud mundial, debido a la alta letalidad de ciertas formas clínicas como la meningitis bacteriana y la septicemia ${ }^{1}$.

El agente causal es Neisseria meningitidis, bacteria aeróbica encapsulada gramnegativa, de la cual se distinguen 14 serogrupos $^{2}$ definidos por los polisacáridos de la cápsula. Seis de ellos son los más patogénicos: A, B, C,W, X e Y. Los serogrupos A y C se han descrito como los de mayor potencial epidémico y últimamente, el serogrupo $\mathrm{W}^{3}$. Éste surge en un brote ocurrido entre los peregrinos del Hajj, en Arabia Saudita, los años 2000 y 2001, diseminándose rápidamente debido a factores como el hacinamiento, el desplazamiento de poblaciones, factores climáticos, conductuales y la virulencia de las cepas circulantes ${ }^{4,5}$.

La EM es de distribución mundial; su reservorio son los seres humanos y se estima que entre 5 y $10 \%$ de la población porta de manera asintomática este agente en el tracto respiratorio superior, especialmente adolescentes y adultos jóvenes ${ }^{6}$. Neisseria meningitidis se transmite de persona a persona a través de secreciones respiratorias por contacto directo (besos) o de aerosoles (tos y estornudos); también se puede transmitir por contacto indirecto a través de utensilios contaminados. Su propagación se ve facilitada por el contacto estrecho y prolongado con una persona infectada, situación que ocurre frecuentemente en internados, dormitorios colectivos o regimientos.

Las principales formas de presentación clínica son: un síndrome meníngeo, septicemia (meningococcemia) y neumonía. Si bien la EM es una enfermedad poco frecuente, su letalidad es de $50 \%$ en casos no tratados y oscila entre 10 y $20 \%$ en aquellos con intervención médica $^{3}$. Causa 50.000 muertes anuales en el mundo, cifra que varía según el grado de desarrollo de los países ${ }^{7}$. En Latinoamérica, las incidencias varían de 0,05/100.000 habitantes en México hasta 1,8/100.000 habitantes en Brasil $^{8}$. La letalidad bajo 5 años de edad es aproximadamente $5 \%$, pudiendo alcanzar a $25 \%$ en adolescentes y adultos ${ }^{9}$. La letalidad es mayor en casos de meningoco-
Facultad de Medicina, Clínica Alemana Universidad del Desarrollo.

Centro de Epidemiología y Políticas de Salud (CEPS) (UDD) (IM, AO, $M N, C G, L P, M H, I D, A M G, X A, G S)$. Ministerio de Salud de Chile (DL, SL, DG).

Directora Consultora Independiente en Epidemiología y Salud Pública Internacional (CIESPI), (EP). Facultad de Medicina, Universidad de Chile. Escuela de Salud Pública (TA). Clínica Alemana Universidad del Desarrollo (UDD) (PV). Hospital Militar, Santiago de Chile (JD).

Los autores explicitan no tener conflicto de interés.

Fuente de financiamiento: Ministerio de Salud de Chile. Licitación pública adjudicada al Centro de Epidemiología y Políticas de Salud de la Facultad de Medicina Clínica Alemana Universidad del Desarrollo,ID757-6-LP13.

Recibido: 10 de marzo de 2015 Aceptado: 6 de octubre de 2015

Correspondencia a: Isabel Matute W. mimatute@udd.c 
ccemia (8-13\%) y puede aumentar a $34-73 \%$ en presencia de shock $^{3}$. El 11 a $19 \%$ de los pacientes que sobreviven presentan secuelas, siendo las más frecuentes la necrosis de extremidades, déficit neurológico y diversos grados de sordera (especialmente en niños) $)^{1,10}$.

Entre los factores descritos en la literatura científica que afectan el curso de la enfermedad y aumentan su letalidad, se encuentran: atención en un hospital de baja complejidad, demora mayor a una hora en el inicio de la terapia antimicrobiana desde el ingreso al hospital en casos graves, reconocimiento tardío de la EM y enfermedades de base del paciente ${ }^{11-16}$.

En Chile, desde 2012 comenzó un aumento de casos de EM sobre lo esperado con características de brote epidémico, a expensas del serogrupo W (58\% del total de casos seroagrupables durante el año 2012) ${ }^{17}$, subiendo la tasa de incidencia de EM de 0,4 por 100.000 habitantes en 2011 a 0,8 en $2012^{18-20}$. Este aumento se acompañó de una letalidad mayor a la observada previamente para la enfermedad en nuestro país, aumentando de 10\% en 2011 a $25 \%$ en $2012^{18,19}$. La presentación clínica se caracterizó por síntomas inespecíficos (compromiso del estado general, fiebre, catarro, vómitos y diarrea, entre otros) y un mayor número de casos con secuelas (amputaciones, hipoacusia o daño neurológico $)^{17}$.

Neisseria meningitidis tiene una alta capacidad de evadir la respuesta inmune mediante variaciones antigénicas de la cápsula y de las proteínas de la membrana externa $^{5,21,22}$, lo cual presenta desafíos profundos para la vigilancia fenotípica y genotípica de las cepas, el control y su prevención ${ }^{23-27}$. Neisseria meningitidis puede adquirir el ADN por transferencia de genes con bacterias de otros serogrupos, dando lugar a la mutación capsular, lo que impide que sean reconocidas por las respuestas inmunitarias del hospedero. De esta forma, el año 2000 surgió una cepa virulenta de $N$. meningitidis serogrupo $\mathrm{W}$, serotipo que si bien no había presentado anteriormente una amenaza grave, causó el gran brote entre los peregrinos del Hajj. El examen genético mostró que una cepa hipervirulenta del serogrupo $C$ había adquirido los genes para la cápsula W a través de la recombinación ${ }^{25,28,29}$. Luego, el 2002, el clon del $\mathrm{W}$ del Hajj provocó una epidemia de meningitis en Burkina Faso y, en 2008, el mismo clon causó una gran proporción de la enfermedad en lactantes en Buenos Aires, Argentina ${ }^{25}$.

En Chile, entre los años 2010 y 2012, el Instituto de Salud Pública (ISP) realizó el análisis genético a un total de 88 cepas de MenW, el que reveló que 95,5\% pertenecía al complejo clonal ST-11 complex/ET37,30, el mismo linaje hipervirulento de las cepas del grupo W que circulan en los últimos años en Argentina y Brasil, relacionadas con las cepas Hajj ${ }^{22,31}$. La características de este complejo clonal, coinciden con lo sucedido en Chile, donde los casos de EM se han manifestado como cuadros graves, principalmente meningococcemias y con una alta letalidad ${ }^{4,30,32}$. Asimismo, el serogrupo W presenta con mayor frecuencia manifestaciones extra-meníngeas asociadas a meningococcemia como infecciones respiratorias o artritis ${ }^{32,33}$. Cualquier retraso puede conducir a una evolución fatal cuando se produce un síndrome séptico, por lo tanto, un diagnóstico preciso y rápido es mandatorio ${ }^{33}$.

En este contexto, la Subsecretaría de Salud Pública del Ministerio de Salud (MINSAL) consideró necesario llevar a cabo un estudio cuyo objetivo fue caracterizar la evolución y desenlace de los casos confirmados de EM el año 2012 y primer trimestre de 2013 en Chile, identificando las características del agente, del hospedero y del proceso de atención médica que pudieron influir en el desenlace de la enfermedad. El estudio fue ejecutado por el Centro de Epidemiología y Políticas de Salud de la Facultad de Medicina Clínica Alemana Universidad del Desarrollo.

\section{Material y Método}

En 2013 se realizó un estudio de serie de casos que incluyó a todos los pacientes confirmados de EM en las regiones con mayor incidencia de casos en el señalado período (3 o más). Estos se identificaron a partir de la base de datos de vigilancia de la EM del Departamento de Epidemiología del MINSAL.

En Chile, esta vigilancia es de notificación obligatoria universal e inmediata frente a la sospecha de casos, los que están definidos para niños sobre un año como fiebre $>38^{\circ} \mathrm{C}$, cefalea y vómitos acompañados de rigidez de nuca o alteración de conciencia o erupción petequial $\mathrm{u}$ otros signos de irritación meníngea. En el lactante bajo un año, se sospecha frente a fiebre con abombamiento de fontanela, vómitos, somnolencia, irritabilidad, convulsiones, con o sin erupción petequial. El caso confirmado se define como el sospechoso que se confirma por laboratorio mediante aislamiento de $N$. meningitidis desde líquido cefalorraquídeo (LCR) u otros fluidos o tejido de sitio normalmente estériles, o por reacción de polimerasa en cadena (RPC) de LCR o plasma ${ }^{34}$. Todas las cepas son confirmadas y serotipificadas en el ISP.

La información para el estudio se obtuvo de la entrevista del caso con un cuestionario estandarizado y de la revisión protocolizada de los registros clínicos. El cuestionario incluyó preguntas sobre posición socio-económica, condiciones de vida, conductas, características físicas e historia médica previa a la EM. También indagó sobre las fases iniciales de la enfermedad y el proceso de atención de salud. En niños bajo 14 años o fallecidos, la encuesta fue respondida por un familiar. Las entrevistas se realizaron entre el 26 de agosto y el 22 de noviembre de 2013.

Las fichas clínicas de los casos fueron facilitadas al 
MINSAL por los Servicios de Salud y establecimientos de atención. La revisión consideró síntomas y valoración clínica al ingreso, tratamiento, complicaciones, secuelas, diagnóstico de egreso, entre otras. Fue llevada a cabo por médicos, salubristas y epidemiólogos entre el 17 de septiembre y el 6 de diciembre de 2013.

Las variables incluidas en ambos instrumentos fueron definidas mediante revisión de literatura científica y consulta a expertos. Las respuestas de la encuesta sobre el proceso de atención y manifestaciones iniciales fueron cotejadas con los registros clínicos.

La información fue digitada en una tableta electrónica usando el programa SurveyToGo y la base de datos se exportó al programa SPSSv21.0 que fue validada semanalmente. Se realizó análisis descriptivo y bivariado según el desenlace (fallecidos y no fallecidos). Para variables cuantitativas se calcularon medidas de tendencia central y de dispersión y para variables categóricas se usaron frecuencias y porcentajes. Se aplicaron pruebas estadísticas para identificar la existencia de diferencias significativas entre casos fallecidos y no fallecidos (considerando un $\mathrm{p}<0,05)$. Finalmente, para evaluar los determinantes de la letalidad, se desarrollaron modelos de regresión logística multivariados que permitieron contar con Odds Ratios (OR) ajustados y su intervalo de confianza (95\%), como estimadores de la probabilidad de morir a partir de la EM.

Se resguardaron aspectos éticos para investigaciones con seres humanos. El protocolo fue aprobado por el Centro de Bioética de Investigación de la Clínica Alemana Universidad del Desarrollo. En la aplicación de cuestionarios y revisión de registros clínicos se utilizaron consentimientos y asentimientos informados, y se desarrolló una estrategia de contención psicológica para familiares de casos fallecidos o con secuelas graves, que incluyó la presencia de un psicólogo durante la entrevista y la derivación a la red de salud correspondiente en casos que lo requirieran. La obtención de registros clínicos por parte del MINSAL se ajustó a la normativa vigente (Art. $4^{\circ}$ del DFL $\mathrm{N}^{\circ} 1$ 2005-MINSAL y Decreto $\mathrm{N}^{\circ} 41$ 2012-MINSAL); además, los ejecutores del proyecto firmaron un acta de confidencialidad de la información contenida en los registros clínicos.

\section{Resultados}

En siete regiones (Antofagasta, Valparaíso, B. O’Higgins, Biobío, Araucanía, Los Lagos y Metropolitana) hubo tres o más casos de EM confirmados en el período, alcanzando un total de 149 casos notificados, que correspondieron a 94,3\% del total de casos del país (n: 158). De ellos, 119 contaron con cuestionario y registro clínico revisados $(79,9 \%), 13$ sólo con ficha clínica, 16 sólo con cuestionario y en un caso no se dispuso de cuestionario ni registro. La tasa de respuesta del cuestionario fue de $90,6 \%(135 / 149)$ y se revisó $88,6 \%$ de los registros clínicos (132/149). Estas diferencias, así como la ausencia de datos en algunas variables (por no respuesta en la encuesta o no registro), implicaron variaciones en algunos denominadores.

\section{Descripción de los casos de enfermedad meningocóccica}

Las características socio-demográficas de los casos analizados fueron: $56,1 \%$ sexo masculino; 21,5 años de edad promedio (mediana $=7,5$ años; rango: 3 meses-93 años), registrándose la tasa de incidencia más alta bajo un año (20,3 por 100.000) (Figura 1); la mayoría residía en la Región Metropolitana $(60,1 \%) ; 8,9 \%$ señaló pertenecer a un pueblo originario y $85,2 \%$ era beneficiario del seguro público de salud (Fondo Nacional de Salud, FONASA) (Tabla 1).

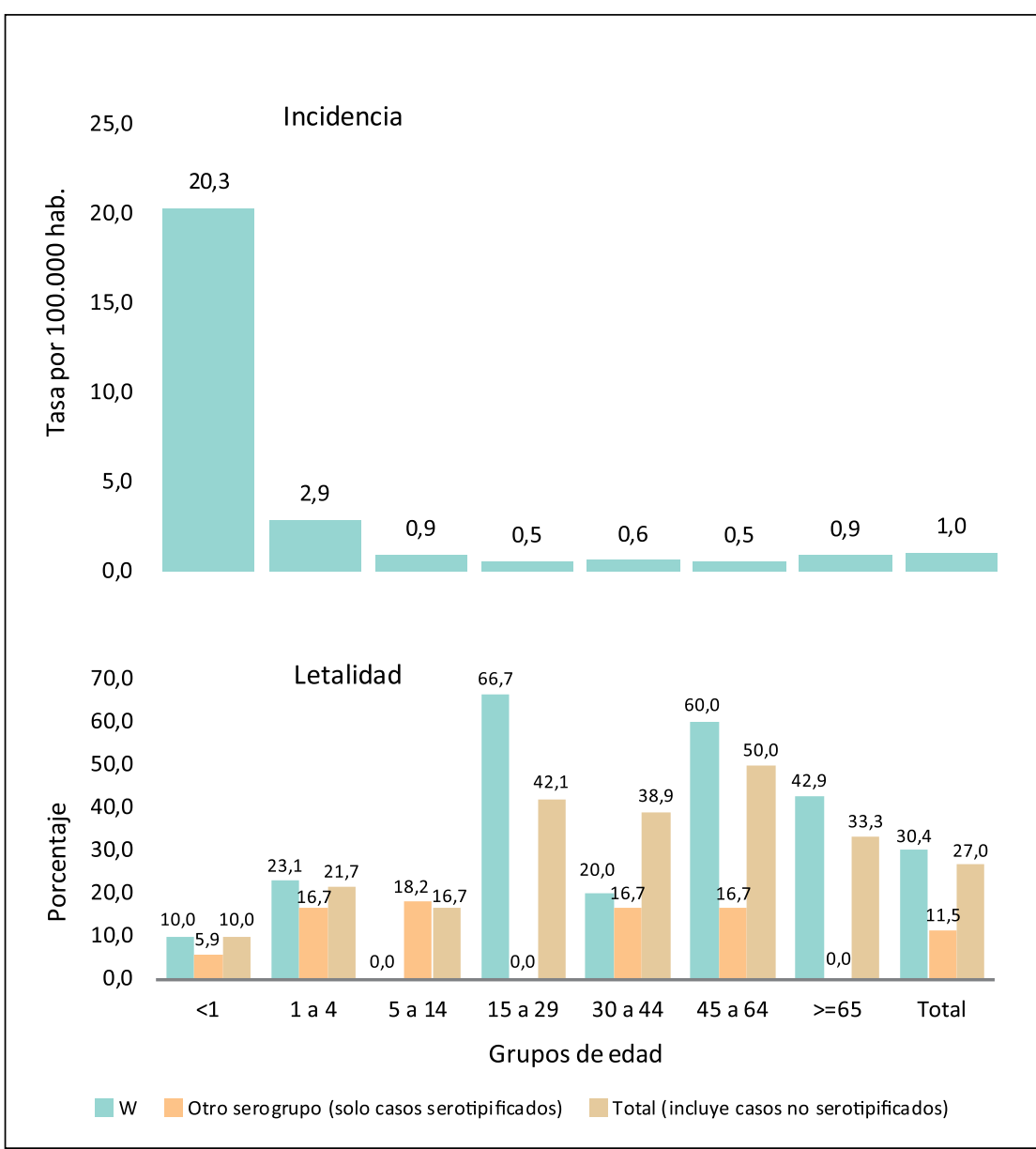

Figura 1. Incidencia y letalidad de EM según grupo de edad. Regiones estudiadas de Chile, enero de 2012- marzo de 2013. 
Tabla 1. Características sociodemográficas de los casos de EM según serogrupo. Regiones estudiadas de Chile, enero de 2012 -marzo de 2013

\begin{tabular}{|c|c|c|c|c|c|c|}
\hline Características* & $\begin{array}{c}\text { B } \\
n: 47\end{array}$ & $\begin{array}{c}\text { W } \\
\text { n. } 69\end{array}$ & $\begin{array}{c}\text { Serogrupo } \\
\text { C eY } \\
\text { n: } 5\end{array}$ & $\begin{array}{l}\mathrm{S} / \mathrm{S}^{* *} \\
\mathrm{n}: 27\end{array}$ & $\begin{array}{c}\text { Total } \\
\mathrm{n}: 148\end{array}$ & Valor $\mathbf{p}^{\dagger}$ \\
\hline Edad (mediana y rango en años) $(n=148)$ & $5(0-73)$ & $7(0-93)$ & $25(0-54)$ & $28(0-75)$ & $7,5(0-93)$ & 0,176 \\
\hline \multicolumn{7}{|l|}{ Sexo $(n=148)$} \\
\hline Hombres & $25(53,2 \%)$ & $43(62,3 \%)$ & $4 \quad(80,0 \%)$ & $11(40,7 \%)$ & $83(56,1 \%)$ & 0,172 \\
\hline Mujeres & $22(46,8 \%)$ & $26(37,7 \%)$ & $1 \quad(20,0 \%)$ & $16(59,3 \%)$ & $65(43,9 \%)$ & \\
\hline \multicolumn{7}{|l|}{ Región de residencia $(n=148)$} \\
\hline Metropolitana & $14(29,8 \%)$ & $56(81,2 \%)$ & $2(40,0 \%)$ & $17(63,0 \%)$ & $89(60,1 \%)$ & $<0,001^{\ddagger}$ \\
\hline Otra región & $33(70,2 \%)$ & $13(18,8 \%)$ & $3(60,0 \%)$ & $10(37,0 \%)$ & $59(39,9 \%)$ & \\
\hline \multicolumn{7}{|l|}{ Pueblo originario $(n=135)$} \\
\hline Pertenece & $1(2,2 \%)$ & $10(16,4 \%)$ & $0 \quad(0,0 \%)$ & $1 \quad(4,2 \%)$ & $12(8,9 \%)$ & $0,049^{\ddagger}$ \\
\hline No pertenece & $45(97,8 \%)$ & $51(83,6 \%)$ & $4(100,0 \%)$ & $23(95,8 \%)$ & $123(91,1 \%)$ & \\
\hline \multicolumn{7}{|l|}{ Nivel educacional en $\geq 6$ años $(n=71)$} \\
\hline Educación básica o inferior & $8(40,0 \%)$ & $14(45,2 \%)$ & $1 \quad(33,3 \%)$ & $4(23,5 \%)$ & $27(38,0 \%)$ & 0,504 \\
\hline Educación media & $9(45,0 \%)$ & $7(22,6 \%)$ & $1 \quad(33,3 \%)$ & $7(41,2 \%)$ & $24(33,8 \%)$ & \\
\hline Educación superior & $3(15,0 \%)$ & $10(32,3 \%)$ & $1 \quad(33,3 \%)$ & $6(35,3 \%)$ & $20(28,2 \%)$ & \\
\hline \multicolumn{7}{|l|}{ Seguro de salud $(n=135)$} \\
\hline Seguro de salud público & $37(80,4 \%)$ & $53(86,9 \%)$ & $3(75,0 \%)$ & $22(91,7 \%)$ & $115(85,2 \%)$ & 0,111 \\
\hline Seguro de salud privado & $6(13,0 \%)$ & $8(13,1 \%)$ & $0 \quad(0,0 \%)$ & $1 \quad(4,2 \%)$ & $15(11,1 \%)$ & \\
\hline FFAA u otro & $3(6,5 \%)$ & $0 \quad(0,0 \%)$ & $1 \quad(25,0 \%)$ & $1 \quad(4,2 \%)$ & $5 \quad(3,7 \%)$ & \\
\hline
\end{tabular}

El 46,6\% de los casos correspondió al serogrupo W, $31,8 \%$ al B, $2,7 \%$ al $\mathrm{C}$ y $0,7 \%$ al Y; sólo $18,2 \%$ no fue serotipificado. El $62,3 \%$ de las personas con serogrupo W fueron hombres y la mediana de edad fue 7 años, con una incidencia bajo un año de 10,2 por 100.000. En este serogrupo se observó mayor probabilidad de residir en la RM y de pertenecer a un pueblo originario (Tabla 1).

\section{Proceso de atención y manejo inicial}

El tiempo transcurrido entre el inicio de síntomas y la primera consulta fue, en promedio, de un día (rango: 0 a 13). El 71,1\% de los pacientes concurrió en primera instancia a servicios de urgencia y 78,9\% fue al sistema público de salud (Tabla 2).

En la primera consulta se sospechó de EM en 8,8\% de los casos, siendo los diagnósticos más frecuentes: infección respiratoria aguda $(28,9 \%, 33$ casos $)$, síndrome febril e infección no especificada $(21,9 \%, 25$ casos), afección gastrointestinal $(15,8 \%, 18$ casos) y exantema $(2,6 \%, 3$ casos). A $14 \%$ no se le informó el diagnóstico (16 casos). Respecto a las indicaciones, a $64 \%$ se lo envió de regreso a su casa, a 19,3\% se lo derivó a otro centro asistencial y $16,7 \%$ fue hospitalizado en la primera atención ( 19 casos). El promedio de consultas realizadas fue de 1,5 (rango: 0 a 6) y $37,6 \%$ de los casos consultó dos o más veces previo a la hospitalización (Tabla 2).

Los síntomas y signos más frecuentes al ingreso hospitalario fueron inespecíficos: fiebre, vómitos, decaimiento, lesiones cutáneas, diarrea y cefalea. Sólo $22,9 \%$ de los casos presentó signos meníngeos al ingreso, proporción que fue menor en el serogrupo W (17,2\%) (Tabla 2). Los signos meníngeos en orden decreciente fueron rigidez de nuca en $21,2 \%$ (25 casos), signo de Brudzinski en $8,5 \%$ (10 casos) y Kernig en 7,6\% (9 casos).

La mayoría de los casos ingresó al hospital como un cuadro infeccioso con foco definido $(55,4 \%)$ y sólo en $22,7 \%$ hubo sospecha diagnóstica de EM al ingreso, la que disminuyó a 14,5\% en el serogrupo W (Tabla 2). Según edad, se sospechó en $28 \%$ de los niños bajo 15 años y en $15,8 \%$ sobre esta edad.

Respecto al diagnóstico de egreso, 51,5\% fue meningococcemia, con diferencias significativas según 
Tabla 2. Presentación clínica de la EM y proceso de atención según serogrupo. Regiones estudiadas de Chile, enero de 2012-marzo de 2013

\section{Características*}

\section{Presentación de signos y síntomas}

Meníngeos (rigidez de nuca, Kernig y/o Brudzinski) $(n=118)$

Fiebre $(n=148)$

Vómitos $(n=148)$

Manchas en la piel $(n=148)$

Diarrea $(n=147)$

Cefalea $(n=148)$

\section{Complicaciones y desenlace}

Complicaciones $(n=132)$

Secuelas $(n=148)$

Letalidad $(n=148)$

\section{Consultas}

$1^{\text {a }}$ consulta en urgencia $(n=114)$

$1^{\text {a }}$ consulta en el sistema público $(n=114)$

Sospecha de EM en $1^{\text {a }}$ consulta $(n=114)$

Envío a domicilio en $1^{\text {a }}$ consulta $(n=114)$

Dos o más consultas previas a hospitalización $(n=133)$

\section{Tratamiento antimicrobiano}

Antimicrobianos en $<$ de 1 día desde inicio síntomas $(n=113)$

Antimicrobianos en $<$ de 1 día desde hospitalización $(n=123)$

\section{Hospitalización}

Hospitalización en el sistema público de salud ( $n=129$ )

Realización de LCR ( $\mathrm{n}=132)$

Realización de hemocultivo $(n=132)$

Infecciones intrahospitalarias $(n=132)$

\section{Sospecha y diagnóstico de ingreso}

Sospecha de EM al ingreso $(n=132)$

\section{Diagnóstico de egreso $(n=132)$}

Meningococcemia (A39.2, A39.3, A39.4)

Meningitis meningocóccica (A39.0)

Waterhouse-Friderichsen (A39.1)

EM sin especificar (A39.9) neuritis retrobulbar)
Decaimiento $(n=148)$

Dg. síndrome infeccioso con foco definido $(n=92)$

Otras localizaciones (artritis, conjuntivitis, encefalitis,

\section{Serogrupo}

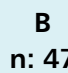

n: 69

$\begin{aligned} 13(31,0 \%) & 10(17,2 \%) \\ 39(83,0 \%) & 64(92,8 \%) \\ 20(42,6 \%) & 46(66,7 \%) \\ 17(36,2 \%) & 25(36,2 \%) \\ 21(44,7 \%) & 14(20,3 \%) \\ 5(10,6 \%) & 28(41,2 \%) \\ 20(42,6 \%) & 14(20,3 \%)\end{aligned}$

5

n: 5

$2(66,7 \%)$

$3(60,0 \%)$

$2(40,0 \%)$

$0 \quad(0,0 \%)$

$0 \quad(0,0 \%)$

$0 \quad(0,0 \%)$

$2(40,0 \%)$
Valor $p^{\dagger}$
$\mathrm{S} / \mathrm{S}$ **

n: 27
Total***

n: 148
$16(37,2 \%)$

$36(52,2 \%)$

$1 \quad(25,0 \%)$

$2(13,3 \%)$

27 (22,9\%)

0,085

$12(44,4 \%)$

$118(79,7 \%)$

$<0,001^{\ddagger}$

$7(25,9 \%)$

$75(50,7 \%)$

$0,002^{\text {\#キ }}$

$4(14,8 \%)$

$46(31,1 \%)$

0,071

$8(29,6 \%)$

$43(29,1 \%)$

$0,017^{\S}$

$7(25,9 \%)$

$40(27,2 \%)$

$0,002^{\S}$

$4(14,8 \%)$

$40(27,0 \%)$

$0,020^{\S \S}$

\section{$7(14,9 \%)$}

$13(18,8 \%)$

$0 \quad(0,0 \%)$

$8(50,0 \%)$

$61(46,2 \%)$

0,361

$5(10,6 \%)$

$21(30,4 \%)$

$1(20,0 \%)$

$1(3,7 \%)$

$21(14,2 \%)$

0,211

$13(48,1 \%)$

$40(27,0 \%)$

$0,005^{\S \S}$

$26(65,0 \%)$

$40(76,9 \%) \quad 0 \quad(0,0 \%)$

$15(71,4 \%)$

$81(71,1 \%)$

0,257

$30(75,0 \%)$

$43(82,7 \%)$

$1(100,0 \%)$

$16(76,2 \%)$

$90(78,9 \%)$

0,977

$4(10,0 \%)$

$2(3,8 \%)$

$0 \quad(0,0 \%)$

$4(19,0 \%)$

$10(8,8 \%)$

$0,026^{\pi}$

$25(62,5 \%)$

$35(67,3 \%)$

$1(100,0 \%)$

$12(57,1 \%)$

$73(64,0 \%)$

0,936

$18(39,1 \%)$

$23(38,3 \%)$

$1 \quad(25,0 \%)$

$8(34,8 \%)$

$50(37,6 \%)$

0,939

$23(54,8 \%)$

$23(40,4 \%)$

$1 \quad(33,3 \%)$

$8(72,7 \%)$

$55(48,7 \%)$

0,391

$33(76,7 \%)$

$48(75,0 \%)$

3 (100,0\%)

$10(76,9 \%)$

$94(76,4 \%)$

0,801

$36(80,0 \%)$

\section{$40(70,2 \%)$}

$3(75,0 \%)$

$20(87,0 \%)$

$99(76,7 \%)$

0,607

$36(83,7 \%)$

$41(59,4 \%)$

$3(75,0 \%)$

$11(68,8 \%)$

$91(68,9 \%)$

0,061

$41(95,3 \%)$

$64(92,8 \%)$

3 (75,0\%)

$14(87,5 \%)$

$122(92,4 \%)$

0,419

$3(7,0 \%)$

$13(18,8 \%)$

$0 \quad(0,0 \%)$

$2(12,5 \%)$

$18(13,6 \%)$

0,278

$13(30,2 \%)$

$10(14,5 \%)$

$1 \quad(25,0 \%)$

$6(37,5 \%)$

$30(22,7 \%)$

0,110

$18(56,3 \%)$

$23(52,3 \%)$

$1(100,0 \%)$

$9(60,0 \%)$

$51(55,4 \%)$

0,150

\begin{tabular}{|c|c|c|c|c|c|}
\hline $16(37,2 \%)$ & $45(65,2 \%)$ & $0 \quad(0,0 \%)$ & $7(43,8 \%)$ & $68(51,5 \%)$ & \multirow{5}{*}{$0,005^{\S}$} \\
\hline $25(58,1 \%)$ & $13(18,8 \%)$ & $3 \quad(75,0 \%)$ & $7(43,8 \%)$ & $48(36,4 \%)$ & \\
\hline $1 \quad(2,3 \%)$ & $5 \quad(7,2 \%)$ & $(0,0 \%)$ & $1 \quad(6,3 \%)$ & $7 \quad(5,3 \%)$ & \\
\hline $1 \quad(2,3 \%)$ & $3(4,3 \%)$ & $1 \quad(25,0 \%)$ & $1 \quad(6,3 \%)$ & $6 \quad(4,5 \%)$ & \\
\hline $0 \quad(0,0 \%)$ & $3(4,3 \%)$ & $(0,0 \%)$ & $0 \quad(0,0 \%)$ & $3 \quad(2,3 \%)$ & \\
\hline
\end{tabular}

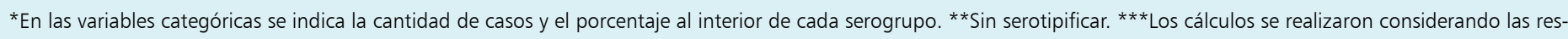

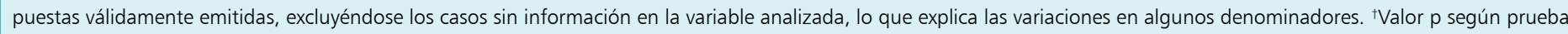

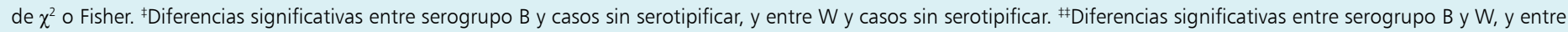

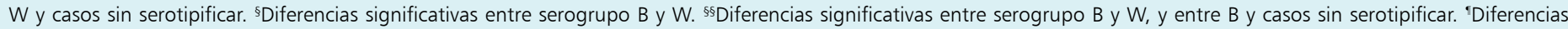
significativas entre serogrupo W y casos sin serotipificar. 
Tabla 3. Cumplimiento de definición de caso de EM según diagnóstico de egreso. Regiones estudiadas de Chile, enero de $2012-$ marzo de 2013

\begin{tabular}{|c|c|c|c|c|c|c|c|c|c|}
\hline \multirow[t]{3}{*}{ Diagnóstico al egreso } & \multicolumn{9}{|c|}{ Cumplimiento de definición de caso de $\mathrm{EM}^{*}$} \\
\hline & & Sí & & & No & & & Total & \\
\hline & $\mathbf{n}$ & $\% \mathrm{col}^{\dagger}$ & $\%$ fila ${ }^{\ddagger}$ & $\mathbf{n}$ & $\% \mathrm{col}^{+}$ & $\%$ fila $^{\ddagger}$ & $\mathbf{n}$ & $\% \mathrm{col}^{+}$ & $\%$ fila $^{\ddagger}$ \\
\hline Meningitis meningocóccica (A39.0) & 23 & 65,7 & 47,9 & 25 & 25,8 & 52,1 & 48 & 36,4 & 100,0 \\
\hline Waterhouse-Friderichsen (A39.1) & 0 & 0,0 & 0,0 & 7 & 7,2 & 100,0 & 7 & 5,3 & 100,0 \\
\hline Meningococcemia (A39.2. A39.3, A39.4) & 12 & 34,3 & 17,6 & 56 & 57,7 & 82,4 & 68 & 51,5 & 100,0 \\
\hline Enf. meningocóccica sin especificar (A39.9) & 0 & 0,0 & 0,0 & 6 & 6,2 & 100,0 & 6 & 4,5 & 100,0 \\
\hline Otras localizaciones** & 0 & 0,0 & 0,0 & 3 & 3,1 & 100,0 & 3 & 2,3 & 100,0 \\
\hline Total & 35 & 100,0 & 26,5 & 97 & 100,0 & 73,5 & 132 & 100,0 & 100,0 \\
\hline
\end{tabular}

$\chi^{2}, p=0,001$. *Considerando los síntomas y signos reportados al ingreso, según definición de caso contenida en la Circular de Vigilancia Epidemiológica y Medidas de Control de Enfermedad Meningocócica del Ministerio de Salud. ${ }^{* *}$ Artritis, conjuntivitis, encefalitis, neuritis retrobulbar. $\%$ col indica la distribución porcentual de los casos con o sin

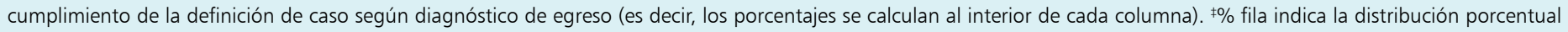
de los casos de cada diagnóstico de egreso según si cumplieron o no la definición de caso (es decir, los porcentajes se calculan al interior de cada fila).

\begin{tabular}{|c|c|c|c|c|c|}
\hline Variable & Fallecidos & No fallecidos & Prob. de fallecer** & $\mathrm{n}^{*}$ & Valor $p$ \\
\hline Edad (años) (伩; DE) & 31,$0 ; 25,4$ & 17,$6 ; 23,8$ & - & $40 / 108$ & $0,002^{\dagger}$ \\
\hline Pertenencia a pueblo originario (\%) & 17,6 & 5,9 & 50,0 & $34 / 101$ & $0,048^{\ddagger}$ \\
\hline Educación media en madres de < 18 años (\%) & 84,6 & 49,3 & 25,0 & $13 / 67$ & $0,042^{\ddagger}$ \\
\hline Enfermedad hipertensiva (\%) & 23,5 & 5,9 & 57,1 & $34 / 101$ & $0,007^{\ddagger}$ \\
\hline Evento estresante (\%) & 64,7 & 40,6 & 35,5 & $34 / 101$ & $0,012^{\ddagger}$ \\
\hline \multicolumn{6}{|c|}{$\begin{array}{l}\text { *n fallecidos/ n no fallecidos. Los cálculos se realizaron considerando las respuestas válidamente emitidas, excluyéndose los casos sin in- } \\
\text { formación en la variable analizada, lo que explica las variaciones en algunos denominadores. }{ }^{*} \text { La probabilidad de fallecer corresponde al } \\
\text { porcentaje de casos fallecidos al interior de cada sub grupo analizado. 'Valor p según prueba Mann-Whitney. }{ }^{\star} \text { Valor p según prueba de } \chi^{2} \text { o } \\
\text { Fisher (variables dicotómicas). }\end{array}$} \\
\hline
\end{tabular}

serogrupo; en el serogrupo $\mathrm{W}$ este diagnóstico alcanzó a $65,2 \%$, bajando en el serogrupo B donde predominó el compromiso meningeo (Tabla 2). Al comparar la sospecha de EM al ingreso y el diagnóstico de egreso contenido en la ficha, sólo se sospechó en 27,1\% de los casos con meningitis (13 casos) y en $19,1 \%$ de los casos con meningococcemia (13 casos).

Al evaluar específicamente la sensibilidad ( $\mathrm{S}=47,9 \%)$ de la definición de caso de meningitis meningocóccica contenida en la Circular de Vigilancia Epidemiológica del Ministerio de Salud de Chile ${ }^{34}$, hubo 52,1\% de casos que siendo confirmados, no cumplían con la definición (Tabla 3).

La mayoría de los casos inició el tratamiento antimicrobiano más de 24 h después del inicio de los síntomas $(51,3 \%)$. Los antimicrobianos más usados dentro de las primeras $72 \mathrm{~h}$ fueron ceftriaxona $(66,7 \%, 88$ casos $)$, cefotaxima $(30,5 \%, 40$ casos) y vancomicina $(25,2 \%, 33$ casos). Respecto al lugar de hospitalización, 76,7\% de los casos lo hizo en el sistema público de salud (Tabla 2).
El 46,2\% de los casos tuvo alguna complicación durante la enfermedad (Tabla 2), siendo las más frecuentes: sepsis $(24,4 \%, 32$ casos), falla renal (19,7\%, 26 casos), shock $(17,6 \%, 23$ casos) y paro cardio-respiratorio $(12,1 \%, 16$ casos $)$. En el serogrupo W, las complicaciones alcanzaron a $52,2 \%$.

Respecto al desenlace, $27 \%$ falleció, 14,2\% sobrevivió con secuelas (neurológicas: 5,4\%, 8 casos; amputaciones: $2,7 \%, 4$ casos; y sensoriales: $2 \%, 3$ casos) y $58,8 \%$ sobrevivió sin registrar secuelas en el corto plazo ( 87 casos). En el serogrupo W la letalidad ascendió a 30,4\% y 18,8\% de los casos sobrevivió con secuelas (Tabla 2).

\section{Determinantes del desenlace de la EM}

Las características de las personas que determinaron mayor probabilidad de fallecer por EM fueron: edad (a mayor edad mayor probabilidad de fallecer) (Tabla 4), pertenencia a pueblos originarios, nivel educacional de la madre en $<18$ años, antecedente de hipertensión arterial $\mathrm{y}$ antecedente de eventos estresantes previos como la 
Tabla 5. Presentación clínica de la EM, exámenes, medidas terapéuticas y complicaciones relacionadas con la probabilidad de fallecer por EM. Regiones estudiadas de Chile, enero de 2012-marzo de 2013

\begin{tabular}{|c|c|c|c|c|c|}
\hline Variable & $\begin{array}{c}\text { Fallecidos } \\
\text { (media y DE) }\end{array}$ & $\begin{array}{l}\text { No fallecidos } \\
\text { (media y DE) }\end{array}$ & Prob. de fallecer* & $\mathrm{n}^{* *}$ & Valor $p$ \\
\hline Frecuencia cardíaca (LPM) al ingreso en $\geq 11$ años & 117,$5 ; 28,4$ & 102,$6 ; 25,4$ & - & $21 / 37$ & $0,045^{\dagger}$ \\
\hline Frecuencia respiratoria (RPM) al ingreso en $\leq 2$ años & 49,$4 ; 13,8$ & 38,$3 ; 12,5$ & - & $7 / 33$ & $0,043^{\dagger}$ \\
\hline Frecuencia respiratoria (RPM) al ingreso en $>2$ años & 31,$8 ; 13,0$ & 26,$0 ; 7,4$ & - & $20 / 37$ & $0,036^{+}$ \\
\hline Presión arterial sistólica $(\mathrm{mmHg})$ al ingreso en $\leq 2$ años & 74,$4 ; 20,9$ & 93,$8 ; 21,4$ & - & $8 / 38$ & $0,024^{+}$ \\
\hline Presión arterial sistólica $(\mathrm{mmHg})$ al ingreso en $>2$ años & 91,$0 ; 31,9$ & 107,$3 ; 25,1$ & - & $24 / 54$ & $0,018^{+}$ \\
\hline Escala de Glasgow (valor más bajo primeras 24 h) & 9,$9 ; 4,7$ & 13,$0 ; 2,7$ & - & $22 / 49$ & $0,005^{\dagger}$ \\
\hline Saturación de oxígeno (\%) al ingreso & 81,$3 ; 21,7$ & 94,$3 ; 7,6$ & - & $27 / 85$ & $<0,001^{\dagger}$ \\
\hline Leucocitos $/ \mathrm{mm}^{3}$ en hemograma & $8.871,2 ; 9.470,5$ & $14.001,3 ; 10.061,6$ & - & 28/92 & $0,008^{+}$ \\
\hline Plaquetas $/ \mathrm{mm}^{3}$ en hemograma & $77.875,1 ; 77.268,6$ & $191.650,2 ; 155.133,6$ & - & $28 / 82$ & $<0,001^{\dagger}$ \\
\hline Creatininemia (mg/dl) & 2,$0 ; 1,9$ & 1,$1 ; 1,6$ & - & $28 / 66$ & $0,001^{+}$ \\
\hline Variable & Fallecidos (\%) & No fallecidos (\%) & Prob. de fallecer* & $\mathrm{n}^{* *}$ & Valor $p$ \\
\hline Diarrea autoreportada & 40,0 & 22,2 & 40,0 & $40 / 108$ & $0,031^{\ddagger}$ \\
\hline Compromiso de conciencia & 42,5 & 17,6 & 47,2 & $40 / 108$ & $0,002^{\ddagger}$ \\
\hline Cambios en coloración de la piel & 17,5 & 6,5 & 50,0 & $40 / 108$ & $0,042^{\ddagger}$ \\
\hline Hipotensión arterial y taquicardia & 10,0 & 0,9 & 80,0 & $40 / 108$ & $0,007^{\ddagger}$ \\
\hline Llene capilar lento al ingreso & 74,1 & 34,2 & 44,4 & $27 / 73$ & $<0,001^{\ddagger}$ \\
\hline Contar con muestra de LCR & 41,2 & 78,6 & 15,4 & $34 / 98$ & $<0,001^{\ddagger}$ \\
\hline Sin indicación de antimicrobiano & 17,6 & 2,0 & 75,0 & $34 / 98$ & $0,002^{\ddagger}$ \\
\hline Sin indicación de cefotaxima & 85,3 & 63,9 & 31,9 & $34 / 98$ & $0,020^{\ddagger}$ \\
\hline Con indicación de corticosteroides & 79,4 & 42,9 & 39,1 & $34 / 98$ & $<0,001^{\ddagger}$ \\
\hline Uso de ventilación mecánica & 85,3 & 34,7 & 46,0 & $34 / 98$ & $<0,001^{\ddagger}$ \\
\hline Presencia de complicaciones & 73,5 & 36,7 & 41,0 & $34 / 98$ & $<0,001^{\ddagger}$ \\
\hline
\end{tabular}

*La probabilidad de fallecer corresponde al porcentaje de casos fallecidos al interior de cada sub grupo analizado. ** $n$ fallecidos/n no fallecidos. Los cálculos se realizaron considerando las respuestas válidamente emitidas, excluyéndose los casos sin información en la variable analizada, lo que explica las variaciones en algunos denominadores. †Valor p según prueba Mann-Whitney. ¥Valor $p$ según prueba de $\chi^{2}$ o Fisher (variables dicotómicas).

muerte de un familiar, divorcio o desempleo, entre otros (Tabla 4).

Respecto al agente causal, las personas afectadas por el serogrupo $\mathrm{W}$ tuvieron una mayor probabilidad de cursar clínicamente con meningococcemia y de fallecer, al ser comparadas con los demás serogrupos (Tabla 1).

Según el diagnóstico de egreso, si bien se observó una mayor probabilidad de fallecer en casos diagnosticados como síndrome de Waterhouse-Friderichsen $(57,1 \%) \mathrm{y}$ como meningococcemia $(29,4 \%)$, estas diferencias no alcanzaron significación estadística.

En aquellos síntomas más inespecíficos al momento de consulta, resultó significativamente asociada a mayor letalidad la presencia de diarrea, compromiso de conciencia y cambios en la coloración de la piel (palidez o piel moteada) (Tabla 5). No fueron significativas las diferencias entre quienes presentaron signos meníngeos y quienes no, falleciendo 26,4 y $22,2 \%$, respectivamente. Por otra parte, en la valoración clínica al ingreso de los pacientes, los signos que mostraron diferencias significativas en el desenlace fueron: mayor taquicardia en $>11$ años (sobre $100 \mathrm{lpm}$ ), mayor taquipnea ( $<2$ años: sobre 40-50 rpm; $>2$ años: sobre $25 \mathrm{rpm}$ ), mayor hipotensión arterial sistólica (<2 años: bajo $90 \mathrm{mmHg}$; > 2 años: bajo $95 \mathrm{mmHg}$ ), menor puntaje de Glasgow y de saturación de oxígeno, llene capilar lento, menor recuento de leucocitos y de plaquetas, y valores de creatininemia más altos (Tabla 5).

En cuanto al proceso de atención, se observó una mayor tendencia a fallecer en quienes consultaron por primera vez en un servicio que no fuese de urgencia y en quienes se hospitalizaron en el sistema público; sin embargo, ninguna de estas diferencias fue estadísticamente significativa. Por otro lado, la mayor letalidad se asoció significativamente con la ausencia de muestra de LCR 


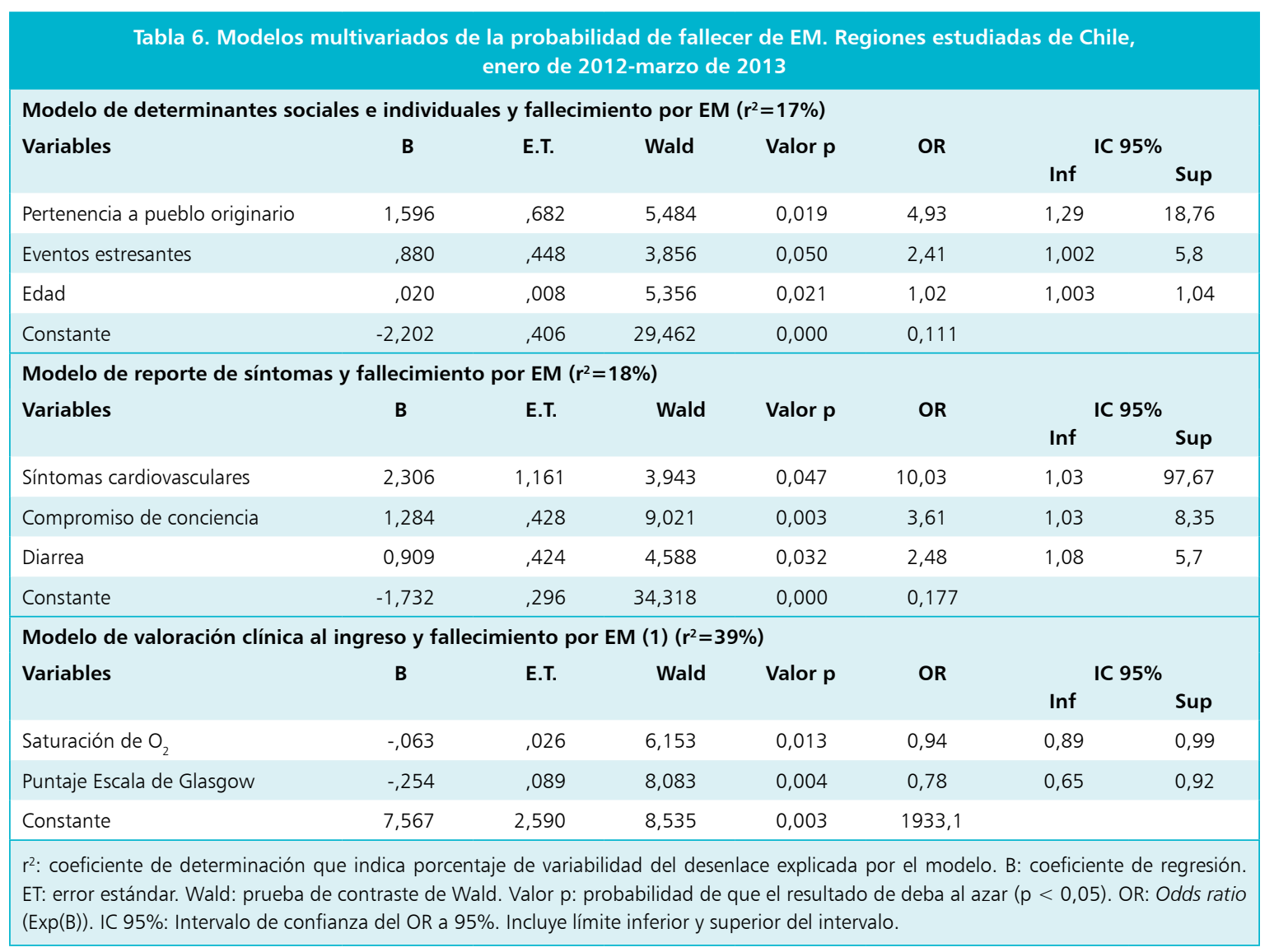

y falta de indicación antimicrobiana, principalmente de cefotaxima; asimismo, fue mayor en quienes recibieron corticosteroides, requirieron ventilación mecánica y presentaron complicaciones (Tabla 5).

No hubo diferencias estadísticamente significativas en cuanto a las horas transcurridas entre las distintas fases de la enfermedad y el proceso de atención y tratamiento; no obstante, en aquellos que fallecieron, el tiempo entre el inicio de síntomas y la primera consulta, fue tres horas mayor; entre el inicio de síntomas y la hospitalización, fue ocho horas mayor y, entre el inicio de síntomas y el comienzo del tratamiento antimicrobiano, también ocho horas superior.

\section{Análisis multivariados}

Al analizar conjuntamente todas las variables estudiadas a través de modelos multivariados, los factores que se mantuvieron asociados a fallecer por EM fueron (Tabla 6):

- Características de los individuos: pertenencia a pueblo originario $(\mathrm{OR}=4,93$; IC95\% $=1,29-18,76)$, haber vivido algún evento estresante en los dos años previos a enfermar $(\mathrm{OR}=2,41$; IC95\% $=1,002-5,8)$ y mayor edad $(\mathrm{OR}=1,02 ; \mathrm{IC} 95 \%=1,003-1,04)$.
- Síntomas al momento de la consulta: síntomas cardiovasculares (hipotensión arterial y taquicardia) $(\mathrm{OR}=10,03$; IC95\% = 1,03-97,67), compromiso de conciencia $(\mathrm{OR}=3,61 ; \mathrm{IC} 95 \%=1,03-8,35)$ y diarrea $(\mathrm{OR}=2,48 ; \mathrm{IC} 95 \%=1,08-5,7)$.

- Valoración clínica al ingreso: mayor saturación de oxígeno $(\mathrm{OR}=0,94$; IC95\% $=0,89-0,99)$ y mayor puntaje de Glasgow $(\mathrm{OR}=0,78 ; \mathrm{IC} 95 \%=0,65-0,92)$ resultaron factores protectores.

En relación al agente causal, si bien en el análisis bivariado las personas con serogrupo $\mathrm{W}$ presentaron mayor probabilidad de fallecer, la significación estadística se perdió en los modelos multivariados.

\section{Discusión}

La letalidad observada en esta serie (27\%) superó las cifras habituales para EM, que varían entre 10 y $20 \%$ en casos con intervención médica ${ }^{3}$. El porcentaje de personas con secuelas $(14,2 \%)$ y el tipo de secuelas registradas coincide con la bibliografía revisada $(11-19 \%)^{1}$, siendo 
las más frecuentes el déficit neurológico, necrosis de extremidades y alteraciones sensoriales.

La letalidad fue mayor en los pacientes con EM por serogrupo W (30,4\%), el que concentró la mayor cantidad de casos del período. Este hallazgo es consistente con lo reportado en la literatura médica sobre el efecto del serogrupo de $N$. meningitidis en la letalidad ${ }^{8}$ y concuerda con la presentación clínica, donde más de la mitad de los casos por serogrupo W debutó con meningococcemia, frente a un tercio en los demás serogrupos, hecho similar a lo reportado en otros brotes ocurridos en el mundo ${ }^{35,36}$. La gran mayoría de los casos de síndrome de Waterhouse Friderichsen también fue causada por el serogrupo W (5 de 7 casos). La letalidad del serogrupo $\mathrm{W}$ fue mayor en todas las edades, pero destaca el grupo de 15 a 29 años (67\%) y el grupo de 45 a 64 años (60\%). Los lactantes bajo un año, a pesar de tener la tasa de incidencia más alta, tuvieron la tasa de letalidad más baja (10\%). Esto también ha sido descrito en estudios internacionales: en Brasil, la letalidad por serogrupo W sobre los 40 años alcanzó a $33,3 \%{ }^{35}$ y en Sudáfrica, $37 \%$ en el grupo de 25 a 44 años $^{36}$.

Las características indagadas en relación a antecedentes del individuo, a la forma de presentación clínica y al proceso de atención médica influyeron en el desenlace fatal, consistentemente con lo reportado por estudios previos $^{37-51}$.

La vulnerabilidad social, expresada en la pertenencia a pueblos originarios ${ }^{37-40}$ y la baja escolaridad materna ${ }^{41}$, se ha vinculado a una mayor probabilidad de morir. En nuestro estudio falleció $50 \%$ de quienes adscribieron a algún pueblo originario, mientras que no se registraron muertes en hijos de madres con educación superior.

Entre las características vinculadas a la susceptibilidad individual encontradas, destaca la edad y haber sufrido eventos estresantes, consistente con lo reportado en la literatura médica ${ }^{42-44}$. Entre los factores no descritos en investigaciones previas, se encontró la presencia de hipertensión arterial que puede atribuirse a la mayor edad y patologías de base de los fallecidos.

Respecto a las características clínicas de los casos, en este estudio la signo-sintomatología que resultó significativa en relación a morir por EM fue: diarrea, compromiso de conciencia, cambios en la coloración de la piel, llene capilar enlentecido e hipotensión arterial y taquicardia; hallazgos descritos también en la literatura médica ${ }^{45-47}$. Algunos estudios muestran que el nivel de conciencia al ingreso puede ser un predictor de sobrevida ${ }^{44}$. En nuestro estudio, $49 \%$ de quienes ingresaron sin respuesta o respuesta sólo al dolor, fallecieron; mientras que aquellos que ingresaron alerta o somnolientos, tuvieron una letalidad de $16 \%$.

De acuerdo al análisis realizado sobre la sensibilidad de la definición de caso de meningitis meningocóccica según las normas del Ministerio de Salud ${ }^{34}$, existe más de $50 \%$ de verdaderos casos que no cumplen dicha definición, lo que la hace poco sensible. Esto debiera motivar una reevaluación de la definición de caso de meningitis, de modo de aumentar la capacidad de sospecha y detección entre los profesionales de atención de salud. Además, se debiera incorporar la definición de meningococcemia dado que su sospecha fue baja $(19,1 \%)$ y tiene una alta probabilidad de ocurrencia cuando se trata de serogrupo W.

En cuanto a los resultados de exámenes, la literatura científica relaciona con mayor letalidad la trombocitopenia y la creatininemia elevada ${ }^{48-50}$, concordantes con los hallazgos de este estudio. Si bien la bibliografía víncula los resultados del $\mathrm{LCR}^{46}$ con la letalidad, en nuestra serie, un bajo porcentaje de casos fallecidos contó con muestra de LCR debido a las malas condiciones hemodinámicas a su ingreso (la toma de muestra de LCR fue significativamente mayor en los sobrevivientes).

En relación al proceso de atención, no se encontraron diferencias estadísticamente significativas en la oportunidad de consulta u hospitalización; tampoco según tipo de establecimiento asistencial, a pesar de que estos factores están ampliamente discutidos en la literatura científica ${ }^{11-16}$.

Respecto a las medidas terapéuticas, una combinación de reconocimiento temprano, traslado rápido al hospital y tratamiento agresivo de las complicaciones mejorarían los resultados del paciente ${ }^{11-16}$. En este estudio, si bien no fueron significativas las diferencias, fue posible constatar un mayor tiempo de latencia entre el inicio de síntomas y la primera consulta, entre el inicio de síntomas y la hospitalización, y entre el inicio de síntomas y el tratamiento antimicrobiano en quienes fallecieron; lo que concuerda con un estudio australiano ${ }^{15}$ que demostró que la demora de $1 \mathrm{~h}(58,7 \mathrm{~min})$ en el inicio de antimicrobianos en casos con EM, desde el ingreso al hospital, estaba asociada directamente con un mal resultado.

En cuanto a los medicamentos, la probabilidad de morir fue mayor en quienes no recibieron terapia antimicrobiana, lo que podría deberse a que aquellos pacientes que ingresaron más graves fallecieron antes de la indicación de tratamiento. Se ha descrito que los casos de septicemia, con el uso precoz de antimicrobianos tienen 2,5 veces menos riesgo de morir, sumado a la administración adecuada de fluidos e inótropos ${ }^{51}$. En la presente serie, destaca la probabilidad tres veces mayor de fallecer en quienes no recibieron una cefalosporina de tercera generación (cefotaxima).

Aquellos casos a los que se les administró algún corticosteroide durante la hospitalización fallecieron en una mayor proporción que los que no recibieron, lo que podría explicarse por la mayor gravedad en quienes tuvieron indicación de corticoterapia. Lo mismo ocurrió con los casos que requirieron ventilación mecánica.

Quienes presentaron complicaciones tuvieron una 
probabilidad significativamente mayor de morir. Respecto a las infecciones asociadas a la hospitalización (respiratorias, tracto urinario, escaras y amputaciones quirúrgicas), los fallecidos tuvieron el doble de infecciones que los sobrevivientes, diferencia no significativa.

Las limitaciones del estudio. Estas se remiten a las dos técnicas utilizadas para el levantamiento de información. En primer lugar, al recoger datos a partir de una encuesta realizada a los casos o familiares, la información fue registrada retrospectivamente, con lo cual puede haberse incurrido en un sesgo de memoria por el tiempo transcurrido y porque se recogió información sobre varios factores de forma simultánea.

Por otra parte, no todos los casos contaban con registro clínico y muchos de ellos eran ilegibles, incompletos, heterogéneos en el registro o denotaban poca acuciosidad en la consignación de los datos. Otra dificultad fue que no se contó con la serotipificación de todos los casos.

Si bien las series de casos carecen de la potencia de otros diseños para probar relaciones causales, la fortaleza de este estudio es la inclusión de prácticamente la totalidad de los casos del período, así como la comparación entre fallecidos y no fallecidos, lo que permitió identificar factores que aumentaron la probabilidad de un desenlace fatal.

En este sentido, considerando el conjunto de la información recabada, es posible concluir que el aumento de la prevalencia del serogrupo $\mathrm{W}$, asociado a la mayor presencia de síntomas inespecíficos o a la rápida progresión a septicemia, impactó en un sistema de salud habituado a cuadros más usuales de EM con localización meníngea, lo que podría explicar en parte, la mayor letalidad observada. Frente a estas circunstancias, el sistema de salud debiera ser capaz de adaptarse a estos nuevos desafíos, con una mejor preparación para enfrentar eventos emergentes y una comunicación de riesgo efectiva.

Agradecimientos. Agradecemos la colaboración de los pacientes y sus familias por su aporte a este proyecto; asimismo, a las SEREMIS de Salud, Servicios de Salud y establecimientos asistenciales que apoyaron con la recolección de datos. También a los profesionales que participaron en diferentes fases del estudio: Dra. Ana María Moraga, EU Maritza García, Ing. Sergio Maass, Geóg. Juan Matute, Soc. Fernando Soto, Dra. Catalina Díaz, Lic. Medicina Valentina Galletti, Bibl. Anita Jazmen y Sec. Antonia Bandera. Finalmente, se agradece especialmente a los expertos cuyo conocimiento contribuyó al desarrollo de esta investigación: Catterina Ferreccio, Francisca Valdivieso, Cecilia Perret, Marcela Potin y Lee Harrison (Pennsylvania, E.U.A.).

\section{Resumen}

Introducción: La enfermedad meningocóccica (EM) es un importante problema mundial por su letalidad y secuelas. Desde 2012 aumentaron en Chile los casos por serogrupo $\mathrm{W}$, con presentación clínica inespecífica, elevada letalidad y secuelas graves. Objetivo: Caracterizar la evolución y desenlace de EM en casos desde enero de 2012 a marzo de 2013 en Chile. Material y Método: Serie de 149 casos de EM de siete regiones. Se aplicó un cuestionario y se revisaron registros clínicos, incluyendo variables del individuo, agente, curso clínico y proceso de atención. Los análisis permitieron obtener OR como estimadores de la probabilidad de fallecer. Resultados: El $51,5 \%$ se presentó como meningococcemia, la letalidad alcanzó a 27\%, predominando el serogrupo W (46,6\%). Aumentaron la probabilidad de fallecer: una mayor edad, pertenencia a pueblos originarios, haber vivido evento estresante, presentar diarrea, compromiso de conciencia, síntomas cardiovasculares, baja saturación de oxígeno y bajo puntaje de Glasgow. Discusión: La letalidad superó las frecuencias habituales y fue mayor en el serogrupo W. El aumento de este serogrupo, asociado a la mayor presencia de síntomas inespecíficos o a la rápida progresión a septicemia, impactó en un sistema de salud habituado a cuadros más clásicos de EM, lo que podría explicar en parte, la mayor letalidad observada.

\section{Referencias bibliográficas}

1.- Pace D, Pollard A J. Meningococcal disease: Clinical presentation and sequelae. Vaccine 2012; 30 (Suppl. 2): B3-9.

2.- Steinhoff M, Nelson K. Global Epidemiology of Meningococcal Infections. En: Nelson $\mathrm{K}$, Master C, editores. Infectious Diseases Epidemiology. Third Ed APHA; 2014. p. 509-17.

3.- Centers for Disease Control and Prevention. Vaccines and Immunizations. Factsheet: Meningococcal Disease and Meningococcal Vaccines. 2009.
4.- Araya P, Díaz J, Seoane M, Fernández J, Terrazas S, Canals A, et al. Vigilancia de laboratorio de enfermedad meningocóccica invasora en Chile, 2006-2012. Rev Chilena Infectol 2014; 31 (4): 377-84.

5.- Harrison L, Jolley K, Shutt K, Marsh J, O’Leary M, Thomson L, et al. Antigenic shift and increased incidence of meningococcal disease. J Infect Dis 2006; 193 (9): 1266-74.

6.- Christensen H, May M, Bowen L, Hickman M, Trotter C. Meningococcal carriage by age: a systematic review and metaanalysis. Lancet Infect Dis 2010;
10 (12): 853-61.

7.- Groves-Pinett M, Abdelnour A, Soley C, Arguedas-Mohs A. Enfermedad meningocócica: epidemiología, diagnóstico y vacunación. Acta Med Costarric 2013; 55 (1): 8-17.

8.- Wilhelm J, Villena R. Historia y epidemiología del meningococo. Rev Chil Pediatr 2012; 83 (6): 533-9.

9.- Gallegos D, Maldonado A, Cáceres K, Seoane M. Situación epidemiológica y cumplimiento de indicadores de la vigilancia de la enfermedad meningocócica. El Vigía MINSAL 2012; 13 (27): 59-63.

10.- Gotuzzo E. Síntomas clínicos de la enfermedad 
meningocócica. En. OPS. Acta del Regional sobre el Meningococo de 2012 Primer Simposio. Buenos Aires, Argentina; 2012. p. 19-20.

11.- Karima T, Bukhari S, Fatani M, Yasin K, Al-Afif K, Hafiz F. Clinical and microbiological spectrum of meningococcal disease in adults during Hajj 2000: an implication of quadrivalent vaccination policy. J Pak Med Assoc 2003; 53 (1): 3-7.

12.- Khan M. Outbreaks of meningococcal meningitis during Hajj: changing face of an old enemy. J Pak Med Assoc 2003; 53 (1): 1-3.

13.- Peltola H, Roine I, Fernández J, Zavala I, Ayala S G, Mata A G, et al. Adjuvant glycerol and/or dexamethasone to improve the outcomes of childhood bacterial meningitis: a prospective, randomized, double-blind, placebo-controlled trial. Clin Infect Dis 2007; 45 (10): 1277-86.

14.- Michael P. Preventing and treating meningococcal meningitis. Med Surg Nurs 2002; 11 (1): 9-12.

15.- Guimont C, Hullick C, Durrheim D, Ryan N, Ferguson J, Massey P. Invasive meningococcal disease-Improving management through structured review of cases in the Hunter New England area, Australia. J Public Health 2010; 32 (1): 38-43.

16.- Donovan C, Blewitt J. An overview of meningitis and meningococcal septicaemia. Emerg Nurse 2009; 17 (7): 30-6.

17.- Moreno G, Vergara N, Gallegos D, Advis M F, Loayza S. Caracterización clínica de los casos de enfermedad meningocóccica por serogrupo W135 confirmados durante el año 2012 en Chile. Rev Chilena Infectol 2013; 30 (4): 350-60.

18.- Ministerio de Salud de Chile. Situación Enfermedad Meningocócica por Serogrupo W-135 Semana Epidemiológica 1 a 52 (desde el 01/01/2012 al 29/12/2012) Plan Acción W-135. 2012.

19.- Ministerio de Salud de Chile. Enfermedad Meninigocócica. Situación Epidemiológica enero-junio 2013. 2013.

20.- Ministerio de Salud de Chile. Situación Enfermedad Meningocócica Semana Epidemiológica 1 a $52(01 / 01 / 2013$ al 24/12/2013) Plan Acción W-135. 2013.

21.- Harrison L. Epidemiological profile of meningococcal disease in the United States. Clin Infect Dis 2010; 50 Suppl 2: S37-44.

22.- López E, Debbag R. Enfermedad meningocóccica: siempre presente. Cambios en los serogrupos en el Cono Sur. Rev Chilena Infectol 2012; 29 (6): 587-94.

23.- Chang Q, Tzeng Y, Stephens D. Meningococcal disease: Changes in epidemiology and prevention. Clin Epidemiol. 2012; 4: 237-45.

24.- Halperin S, Bettinger J, Greenwood B, Harrison L, Jelfs J, Ladhani S, et al. The changing and dynamic epidemiology of meningococcal disease. Vaccine 2012; 30 (Suppl. 2).

25.- Harrison L. La epidemiología mundial de la enfermedad meningocócica. En. OPS. Acta del Regional sobre el Meningococo de 2012. Primer Simposio. Buenos Aires, Argentina; 2012. p. 4-5.

26.- De Olivera L. La importancia de la vigilancia de buena calidad para el reconocimiento de la carga de morbilidad meningocócica. En: OPS. Acta del Regional sobre el Meningococo de 2012 Primer Simposio. Buenos Aires, Argentina; 2012. p. 11-2.

27.- Ibarz A. El programa SIREVA en América Latina: mejoramiento de la vigilancia y tipificación de la enfermedad meningocócica en América Latina y el Caribe. En: OPS. Acta del Regional sobre el Meningococo de 2012. Primer Simposio. Buenos Aires, Argentina; 2012. p. 14-5.

28.- Castiñeiras T, Barroso D, Marsh J, Tulenko M, Krauland M, Rebelo M, et al. Capsular switching in invasive Neisseria meningitidis, Brazil. Emerg Infect Dis 2012; 18 (8): 1336-8.

29.- Prifert C, Streng A, Krempl C, Liese J, Weissbrich B. Travel-related Neisseria meningitidis serogroup W135 infection, France. Emerg Infect Dis 2013; 19 (6): 1030-2.

30.- Valenzuela M, Moreno G, Vaquero A, Seoane M, Hormazábal J, Bertoglia M, et al. Emergencia de la cepa W135 causante de enfermedad meningocócica invasora en Chile 2012. Rev Med Chile 2013; 141: 959-67.

31.- Izquierdo G, Villena R. Enfermedad meningocócica: epidemiología y vacunas, un enfoque práctico. Rev Médica Clínica Las Condes. Elsevier 2014; 25 (3): 541-6.

32.- Rosas R, Solar S, Durán L, Porte L, Noriega L, Thompson L, et al. Infecciones respiratorias bacteriémicas por Neisseria meningitidis serogrupo W. Rev Chilena Infectol 2015; 32 (2): $242-3$.

33.- Vienne P, Ducos-Galand M, Guiyoule A, Pires R, Giorgini D, Taha M, et al. The role of particular strains of Neisseria meningitidis in meningococcal arthritis, pericarditis, and pneumonia. Clin Infect Dis 2003; 37 (12): 1639-42.

34.- Ministerio de Salud de Chile. Circular B51 $\mathrm{N}^{\circ} 09$. Vigilancia epidemiológica y medidas de control de enfermedad meningocóccica (A39). 2009.

35.- Masuda E, Marques T, Borelli R, Casagrande S, Susuki P, Waldman E. Mortalidade por doença meningocócica no Município de São Paulo, Brasil: características e preditores. Cad Saúde Pública 2015; 31 (2): 405-16.

36.- Von Gottberg A, du Plessis M, Cohen C, Prentice E, Schrag S, de Gouveia L, et al. Emergence of endemic serogroup W135 meningococcal disease associated with a high mortality rate in South Africa. Clin Infect Dis 2008; 46 (3): 377-86.

37.- Alvarado A, Castillo L. Meningitis bacteriana (Revisión bibliográfica). Rev Med Leg Costa Rica 2006; 23 (1): 129-42.

38.- Daley A. Meningococcal disease. Aust Fam Physician 2003; 32 (8): 597-605.

39.- Simmons G, Martin D, Stewart J, Jones N, Calder L, Bremner D. Carriage of Neisseria meningitidis among household contacts of patients with meningococcal disease in New Zealand. Eur J Clin Microbiol Infect Dis 2001; 20: 237-42.

40.- Baker M G, Martin D R, Kieft C E, Lennon D. A 10-year serogroup B meningococcal disease epidemic in New Zealand: descriptive epidemiology, 1991-2000. J Paediatr Child Health 2001; 37: S13-9.

41.- Kriz P, Bobak M, Kriz B. Parental smoking, socioeconomic factors and risk of invasive meningococcal disease in children: a population based case-control study. Arch Dis Child 2000; 83: 117-21.

42.- Cohn A, MacNeil J, Harrison L, Hatcher C, Theodore J, Schmidt M, et al. Changes in Neisseria meningitidis disease epidemiology in the United States, 1998-2007: implications for prevention of meningococcal disease. Clin Infect Dis 2010; 50 (2): 184-91.

43.- Kaplan S, Schutze G, Leake J, Barson W, Halasa N, Byington C, et al. Multicenter surveillance of invasive meningococcal infections in children. Pediatrics 2006; 118 (4): e979-84.

44.- Nørgård B, Sørensen H, Jensen E, Faber T, Schønheyder H, Nielsen G. Pre-hospital parenteral antibiotic treatment of meningococcal disease and case fatality: a Danish population-based cohort study. J Infect 2002; 45: 144-51.

45.- Casella E, Cypel S, Osmo A, Okay Y, Helena B, Lichtig I, et al. Sequelae from meningococcal meningitis in children. A critical analysis of dexamethasone therapy. Arq Neuropsiquiatr 2004; 62: 421-8.

46.- Smith I, Bjørnevik T, Augland I, Berstad A, Wentzel-Larsen T, Halstensen A. Variations in case fatality and fatality risk factors of meningococcal disease in Western Norway, 1985-2002. Epidemiol Infect 2006; 134: 103-10. 
47.- Heckenberg S, de Gans J, Brouwer M, Weisfelt M, Piet J, Spanjaard L, et al. Clinical features, outcome, and meningococcal genotype in 258 adults with meningococcal meningitis: a prospective cohort study. Medicine 2008; 87 (4): $185-92$.

48.- Emonts M, Sweep F, Grebenchtchikov N, Geurts-Moespot A, Knaup M, Chanson A, et al.
Association between high levels of blood macrophage migration inhibitory factor, inappropriate adrenal response, and early death in patients with severe sepsis. Clin Infect Dis 2007; 44: 1321-8.

49.- Herrera M, Rojas A, Izaguirre J, Casanova L. Guías de manejo clínico: Consenso de Meningitis. Diagnóstico. Arch Venez Pueric
Pediatr 2010; 73: 37-44.

50.- Ministerio de Sanidad Servicios Sociales e Igualdad de España. Guía de Práctica Clínica sobre el Manejo de la Enfermedad Meningocócica Invasiva. 2013.

51.- Sabra A, Benger J. Meningococcal disease in children: a clinical review. Turk J Pediatr 2011; 53: 477-88. 